\title{
Desenvolvimento de guias alimentares em diversos países
}

\author{
Development of food-based dietary \\ guidelines in several countries
}

Roseane Moreira Sampaio BARBOSA'

Luciléia Granhen Tavares COLARES²

Eliane de Abreu SOARES 2,3

\section{RE S U M O}

Os guias dietéticos baseados nos alimentos têm objetivo de favorecer a educação nutricional, utilizando termos que sejam compreensíveis, simples e claros para a maioria dos consumidores e indicando as modificações necessárias nos padrões alimentares. Este trabalho foi realizado com o objetivo de apresentar uma revisão da literatura sobre o desenvolvimento dos guias alimentares em vários países. Para tanto, foi realizado um levantamento bibliográfico no período de 1991 a 2006 nas bases de dados Medline e Lilacs, utilizando como palavras-chave "guia alimentar", "guia dietético" e "desenvolvimento de guia dietético" com o intuito de aprofundar os seguintes itens: elaboração, avaliação, implantação e problemas metodológicos relacionados ao desenvolvimento dos mesmos. Desde 1996, vêm sendo publicadas orientações pelo Comite de Alimentação e Nutrição/Organização Mundial de Saúde para elaboração dos guias alimentares e vários países os desenvolveram de acordo com essas recomendações, apesar dos diversos problemas metodológicos enfrentados. Após o desenvolvimento dos guias alimentares, os maiores desafios são a implantação e a avaliação. Dentre as estratégias para sua divulgação destaca-se a elaboração de ferramentas de educação nutricional, tais como: representação gráfica, folder, apostila e pôster. Com relação à avaliação dos guias alimentares, poucos países realizaram pesquisas abordando o conhecimento e o entendimento dos consumidores. Conclui-se que o processo de desenvolvimento dos guias alimentares é complexo e é necessário realizar um planejamento para sua implantação e avaliação, esta última devendo se voltar tanto ao entendimento como ao impacto sobre a saúde da população.

Termos de indexação: Educação alimentar e nutricional. Guias alimentares. Nutrição em saúde pública.

\section{A B S T R A C T}

Food-based dietary guidelines have the objective of favoring nutrition education using terms which are understandable, simple and clear for most consumers, indicating the necessary modifications in food patterns.

\footnotetext{
1 Universidade Federal do Rio de Janeiro, Curso de Nutrição Humana. Av. Brigadeiro Trompowsky, s/n., Cidade Universitária, Ilha do Fundão, 21949-900, Rio de Janeiro, RJ, Brasil. Correspondência para/Correspondence to: R.M.S. BARBOSA. E-mail: <roseanesampaio@ig.com.br>

2 Universidade Federal do Rio de Janeiro, Instituto de Nutrição Josué de Castro. Rio de Janeiro, RJ, Brasil.

${ }^{3}$ Universidade do Estado do Rio de Janeiro. Instituto de Nutrição. Rio de Janeiro, RJ, Brasil.
} 
This work was conducted with the aim of presenting a review of the literature on developing dietary guidelines in many countries. In order to accomplish this, a bibliographical survey of the period from 1991 to 2006 of Medline and Lilacs databases was performed using "food guide", "dietary guideline" and "development of dietary guideline" as keywords with the intention of carrying out an in-depth research of the following items: preparation, evaluation, implementation and methodological issues related to their development. Since 1996, orientations given by the Food and Nutrition Board/World Health Organization have been published for preparation of dietary guidelines and many countries developed them in accordance to these recommendations, despite facing numerous methodological issues. After dietary guidelines are developed, the biggest challenge is to implement and evaluate them, and among the strategies for their dissemination, the development of nutrition education tools such as graphs, folders, printed leaflets and posters is pointed out. Regarding the assessment of food guides, few countries performed researches that focused on consumer knowledge and understanding. We concluded that the process of developing dietary guidelines is very complex and it is necessary to plan their implementation and evaluation, not only regarding their understanding by consumers but also their impact on population health.

Indexing terms: Food and nutrition education. Food guide. Nutrition, public health.

\section{N T R O D U ÇÃ O}

Atualmente, as mensagens nutricionais para a população são consideradas um desafio, pois os consumidores são constantemente bombardeados pela mídia com diversas informações que se distanciam de condutas saudáveis ${ }^{1}$.

Na Conferência Internacional de Nutrição, realizada em Roma em 1992, foram identificadas estratégias e ações para melhorar o consumo alimentar e o bem-estar nutricional da população. De acordo com este evento deve-se estimular a elaboração de guias alimentares para diferentes grupos etários e, para atingir este objetivo, cada país deve planejar ações de acordo com sua cultura e com os problemas de saúde relacionados à alimentação ${ }^{2}$. Segundo Anderson \& Zlotkin ${ }^{3}$ os guias alimentares serão efetivos se contribuírem para promover mudança no consumo dietético. Caso sejam elaborados sem o conhecimento da prevalência de morbimortalidade de determinada população, provavelmente não terão sucesso.

Os guias alimentares oficiais de diferentes países possuem diversos formatos, diferentes números de grupos alimentares e de porções, porém eles têm o mesmo objetivo, transformar o conhecimento científico de nutrição em conceitos básicos para que grande parcela da população seja orientada quanto à forma de se alimentar adequadamente ${ }^{4}$.

Diversos países vêm desenvolvendo seus guias alimentares, mas são escassos os estudos que abordem a metodologia utilizada para sua elaboração, implantação e avaliação. Portanto, este trabalho foi realizado com o objetivo de apresentar uma revisão da literatura sobre a elaboração dos guias alimentares em vários países. Para tanto, foi realizado um levantamento bibliográfico no período de 1991 a 2006 nas bases de dados MEDLINE e Lilacs, utilizando como palavras-chave "guia alimentar", "guia dietético" e "desenvolvimento de guia dietético" empregadas no idioma inglês com o intuito de aprofundar os seguintes itens: desenvolvimento, avaliação, implantação e problemas metodológicos relacionados ao desenvolvimento de guias alimentares.

\section{Elaboração dos guias dietéticos baseados nos alimentos}

De acordo com a Food and Nutrition Board/World Health Organization (FNBMHO) ${ }^{5}$, os guias dietéticos baseados nos alimentos (Food Based Dietary Guidelines - FBDG) têm como objetivo favorecer a educação nutricional a partir de termos que sejam compreensíveis, simples e claros para a maioria dos consumidores, e que indiquem as modificações necessárias nos padrões alimentares de uma dada população rural e urbana e mesmo de grupos específicos.

Calderón \& Morón ${ }^{6}$ destacaram a importância do desenvolvimento dos guias alimentares baseados nos alimentos em lugar daqueles ba- 
seados em nutrientes, na promoção de estilo de vida saudável e na prevenção de doenças.

A principal razão para o desenvolvimento dos FBDG é o fato de que informações baseadas nos alimentos são de mais fácil compreensão do que as baseadas nos nutrientes, porém alguns aspectos como o padrão dietético, a praticidade, a flexibilidade, a compreensão e a aceitação cultural devem ser considerados ${ }^{5}$.

Para o desenvolvimento dos FBDG destacam-se cinco etapas: 1) identificação dos problemas de saúde relacionados com a dieta estimando a magnitude desses problemas e estabelecendo as prioridades; 2) avaliação dos padrões de consumo alimentar coletados a partir de diferentes métodos dietéticos; 3) integração dos FBDG às políticas e programas nacionais de saúde e alimentação; 4) construção do guia com objetivo de prevenir déficit ou excesso de energia, incluindo a adequação de macronutrientes, promover um aporte adequado de vitaminas e minerais, bem como ressaltar a importância da atividade física e 5) avaliação da aceitação do guia e de sua representação visual para que se processe a divulgação ao público alvo por diversos meios de comunicação ${ }^{5}$.

As etapas para a preparação dos FBDG propostas por Caldeirón \& Morón ${ }^{6}$ se assemelham àquelas propostas pela $\mathrm{FNB} / \mathrm{NHO}^{5}$. Ambos descrevem que as mensagens devem ser diretas e positivas, apontando os benefícios de uma alimentação saudável e levando em consideração o nível de escolaridade da população. Devem-se também investigar a percepção, as crenças, expectativas e os valores a respeito dos alimentos, da nutrição e da alimentação saudável dos diferentes segmentos da população. Outro aspecto apontado pelos autores é o conhecimento da prevalência e da incidência de deficiência de micronutrientes, da freqüência e do consumo de alimentos de baixa qualidade nutricional e a possibilidade da existência de contaminantes químicos e biológicos nos alimentos.

Em 2004, a Society for Nutrition Education $(\mathrm{SNE})^{7}$ também fez algumas recomendações para a elaboração do guia alimentar para os Estados Unidos da América (EUA), como: estar de acordo com as recomendações dietéticas americanas; possibilitar a aprendizagem e as mudanças de comportamento alimentar; divulgar os conceitos básicos da nutrição (variedade, proporcionalidade e moderação); estabelecer as porções de cada grupo alimentar podendo variar (para mais ou para menos) para diferenciar o consumo energético e enfatizar a prática regular de atividade física. Esta instituição apóia o uso da representação gráfica como ferramenta de educação nutricional ${ }^{7}$.

A representação gráfica do guia alimentar tem o propósito de ajudar o grupo populacional eleito a recordar facilmente que alimentos devem ser incluídos na dieta e em que proporções. A construção de um símbolo de fácil compreensão que represente o guia serve como excelente ferramenta de educação nutricional e pode ser utilizada por consumidores, por profissionais de educação nutricional e pela indústria de alimentos ${ }^{7,8}$.

O primeiro guia alimentar de que se tem conhecimento foi proposto em 1916 nos EUA, por Caroline Hunt, que recomendou uma alimentação saudável por meio de mensagens, sendo depois desenvolvidas diversas representações gráficas. A maioria dessas figuras apresentava-se no formato circular, mas com um número diferente de grupos alimentares ${ }^{9,10}$. Posteriormente, alguns países decidiram modificar o formato do guia alimentar seguindo o guia norte-americano (pirâmide), com exceção de alguns países como Canadá, que desenvolveu o arco-íris, Costa-Rica, o formato de uma pizza, Guatemala, que optou pela representação gráfica de um pote de cerâmica e o México, que adotou a figura da maçã. Já os países da Europa optaram pela pirâmide e pelo círculo ${ }^{11,12}$.

Alguns países realizaram pesquisas para selecionar a melhor representação gráfica do guia alimentar. A United States Department of Agriculture (USDA) realizou extensa pesquisa em três etapas para decidir a representação gráfica para seu país. Na primeira etapa (1981-1984) nutricionistas selecionaram os objetivos dietéticos e definiram os grupos alimentares calculando o número de 
porções diárias dando origem à Roda dos Alimentos. Como o desenho da roda não representava a moderação e a proporcionalidade, a USDA continuou utilizando somente os grupos alimentares e o tamanho das porções nas suas publicações. A segunda etapa teve início em 1988, na qual várias representações gráficas foram avaliadas tendo a participação de adultos com nível médio de escolaridade. Pesquisas que utilizaram discussões com grupos focais indicaram que os grupos alimentares deviam ser mostrados em formato de triângulo (pirâmide), por refletirem melhor os conceitos nutricionais: moderação, proporcionalidade e variedade ${ }^{13}$.

A terceira etapa da elaboração do guia americano se iniciou em 1991, depois que a USDA publicou a Pirâmide Alimentar. Uma empresa de consultoria (Bells Associates) utilizou o método de pesquisa social, grupo focal com representantes da indústria de alimentos e nutricionistas, para comparar a pirâmide com outros ícones com o formato de tigela, maçã e carrinho de compras. Essa pesquisa mostrou que os representantes da indústria preferiam o formato da maçã e da tigela e o grupo dos nutricionistas deu preferência à pirâmide por achar que transmitia melhor as mensagens desejadas. Nesse momento a pesquisa passou a avaliar apenas dois formatos, a pirâmide e a tigela, sendo coletadas opiniões de 3 mil crianças e adultos de baixa renda. Os resultados indicaram que ambas as representações gráficas transmitiram a necessidade de consumir uma alimentação com variedade, mas a pirâmide meIhor indicou a moderação e a proporcionalidade ${ }^{13}$.

Mesmo depois de publicada, a pirâmide recebeu críticas que continuam estimulando debates. Os produtores de carne, leite, e alimentos processados queixaram-se que o desenho geométrico da pirâmide evidencia uma hierarquia dos alimentos que, ao orientar para um consumo moderado, podia diminuir a venda de seus produtos. Outra crítica diz respeito à estrutura conceitual, às bases científicas e à efetividade da pirâmide como ferramenta de educação nutricional ${ }^{13}$. De acordo com Achterberg ${ }^{14}$, alguns estudos que avaliaram os guias alimentares mostraram que a pirâmide americana não era compreendida pelos consumidores. Eles não entendiam se os grupos de alimentos que estão no topo ou na base da pirâmide eram os mais importantes. E consideram que as gotas brancas de gorduras e açúcar espaIhadas na pirâmide eram defeitos na impressão, além de não entenderem o conceito de porção.

Na Inglaterra, Hunt et al. ${ }^{11}$ selecionaram o melhor formato do guia alimentar Britânico a partir de um estudo que envolveu 2074 indivíduos e 230 profissionais que trabalhavam com educação nutricional. Nessa pesquisa foram avaliados possíveis formatos das representações gráficas para o guia nacional. Foram testados vários formatos (pirâmide, triângulo, prato, arco-íris e círculo). A maioria dos indivíduos entendeu que todos esses formatos representavam adequadamente a proporção dos grupos alimentares e o conceito de uma dieta saudável, mas a preferência foi pelo formato do círculo, particularmente o prato.

Em 1996, o Nutrition Institute of the State Baja Califórnia (IUNBAC) desenvolveu um guia alimentar regional no formato de maçã "Maçã Saudável"15. Em 1998, o National Institute of Nutrition desenvolveu o seu guia alimentar "Pirâmide da Saúde" para a população mexicana ${ }^{16}$.

Em 2002, Bacardi-Gascon et al..$^{16}$ avaliaram esses dois guias alimentares desenvolvidos na Califórnia e no México, por meio de grupos focais com mulheres de dois níveis de escolaridade (7 e 15 anos de escolaridade), para comparar os dois formatos, a maçã e a pirâmide. A proposta da pesquisa foi avaliar e comparar a representação gráfica, a compreensão das mensagens e a habilidade de aplicar ambos os guias. Foram desenvolvidos exercícios para planejamento de dieta de um dia com base nos guias, com o objetivo de avaliar o nível de compreensão dos consumidores quando utilizavam cada guia isoladamente. Os resultados mostraram que o percentual médio do escore de planejamento da dieta obtido pelos participantes utilizando a "Pirâmide da Saúde" foi de 64,1 e usando a "Maçã da Saúde" foi de 
76,7. Embora não tenha havido diferença estatisticamente significante, o guia em formato de maçã foi o preferido pelas participantes, por ser mais atrativo, colorido e fornecer de forma mais clara a informação sobre os alimentos, isto é, a identificação dos alimentos e o número de porções de cada grupo alimentar. A pesquisa recomendou a "Maçã Saudável" como ferramenta de educação nutricional para a população da Califórnia.

A Roda dos Alimentos, elaborada em Portugal no final da década de 70, representada graficamente por um círculo subdividido em fatias de diferentes tamanhos, que apresentava os alimentos com propriedades nutricionais semelhantes, foi um instrumento inovador utilizado por diversos países como material educativo. Teve como objetivo transformar informações nutricionais complexas em conceitos simples e fáceis de serem utilizados pela população leiga e atendeu à realidade alimentar da época. Passados praticamente 25 anos do seu lançamento surgiram a necessidade e a oportunidade de atualizar esse instrumento por meio de uma parceria entre a Faculdade de Ciências da Nutrição da Universidade do Porto e o Instituto do Consumidor ${ }^{17}$.

Em 2003 foi desenvolvido o novo guia alimentar português elaborado em nove etapas: $1^{\text {a) }}$ obtenção da opinião de pesquisadores sobre alimentação e nutrição; $2^{a}$ ) estabelecimento dos objetivos nutricionais, isto é, a recomendação de energia de 13 grupos populacionais de ambos os sexos (crianças acima de 1 ano até adultos), a distribuição de macronutrientes, micronutrientes e de fibras alimentares; $3^{\mathrm{a}}$ ) definição dos 7 grupos e 21 subgrupos de alimentos de acordo com composição nutricional, hábitos alimentares dos portugueses em substituição aos 5 grupos já utilizados na primeira versão da roda de alimentos ${ }^{17}$.

A 4 a etapa do guia português foi o estabelecimento da porção padrão de cada grupo alimentar, sendo baseada na média de peso de medidas caseiras (copo, colher de sopa) ou em unidades (um ovo, um pão). Na $5^{\text {a }}$ etapa estabeleceu-se a porção de equivalente de cada grupo alimentar e na $6^{\text {a }}$ etapa definiu-se o número recomendado de porções diárias de cada grupo alimentar, com base em três dietas calculadas de acordo com o valor energético total de 1300, 2200 e $3 \mathrm{mil} / \mathrm{kcal}$. Na $7^{\mathrm{a}}$ etapa observou-se o fornecimento de alguns componentes dietéticos, pelo cálculo das três dietas e comparou-se com as recomendações. Somente os valores de sódio e iodo não atenderam às recomendações ${ }^{17}$.

A $8^{a}$ etapa consistiu na apresentação dos resultados e o ícone escolhido foi o formato do círculo por estar associado à imagem de um prato e por já ser reconhecido pelos indivíduos por meio da primeira versão do guia alimentar. O prato é um importante símbolo da cultura portuguesa, pois a mesa é um lugar comum e importante para as refeições. De acordo com os autores os grupos de alimentos apresentados no formato do círculo mostram a mesma importância e visualmente se complementam, ao contrário da pirâmide, que representa a idéia hierarquizada dos grupos alimentares. A última etapa foi a divulgação do material por folder para a autoridade de saúde, comunidade científica, para a mídia e a internet ${ }^{17}$. De acordo com Rodrigues et al. ${ }^{17}$, apesar da falta de informação a respeito de qual metodologia de pesquisa foi utilizada no guia dietético anterior, observa-se que as porções recomendadas de cada grupo alimentar na Nova Roda de Alimentos, não diferem muito do guia alimentar anterior, desenvolvido em 1977. Os autores relataram a necessidade de atualização das recomendações de energia e nutrientes para a população portuguesa, pois esses dados são de 1982. Além disso, as tabelas de composição química dos alimentos foram publicadas em 1985. Outro problema e, provavelmente, o mais relevante, deve-se ao fato de que a última pesquisa de consumo alimentar em Portugal foi realizada em 1980, o que pode não retratar o atual padrão dietético da população.

A Tailândia também desenvolveu seu guia alimentar, cuja representação gráfica é o formato de uma bandeira, "A Bandeira Nutricional", sendo uma versão invertida da Pirâmide da USDA. O maior grupo é representado pelos alimentos ricos 
em amido que estão no topo, e no vértice estão incluídos os alimentos do grupo do sal, açúcar e óleo. Há uma variação do número de porções dependendo do valor energético total da dieta, sendo de 1 600, 2000 e 2 400kcal ${ }^{1}$.

Depois de se tornar o guia oficial para a população da Tailândia, ele foi testado com outro símbolo, o leque, utilizando o método teste local central (central location testing - CLT). O CLT é uma técnica de pesquisa na qual os pesquisadores realizam o estudo em locais freqüentados pelo grupo a ser pesquisado. Os cinco locais selecionados foram as universidades, as lojas de departamentos, as fábricas, os supermercados e os pontos de ônibus, com o objetivo de testar o guia alimentar mais apropriado, o entendimento dos conceitos nutricionais, e determinar a preferência entre dois símbolos (bandeira e leque). Os resultados demonstraram que os consumidores confundiram a palavra "porção" no grupo das frutas porque elas não podem ser medidas com a utilização de utensílios. Aproximadamente $60 \%$ dos participantes não entenderam a variação das porções de cada grupo alimentar, devido ao ajuste de acordo com a necessidade energética. Os consumidores preferiram a bandeira ao leque, por ela ser de mais fácil entendimento e determinar a quantidade recomendada de cada grupo alimentar ${ }^{1}$.

Na África do Sul foi realizada pesquisa e, por meio de grupos focais, foram avaliadas a compreensão de 10 mensagens com a representação gráfica do guia alimentar. Os resultados mostraram que as mensagens eram bem compreendidas pelos consumidores e que a representação gráfica do guia alimentar, como complemento, não era necessária'.

Em vários países como África do Sul, União Européia, Espanha e Brasil o processo de desenvolvimento dos guias alimentares baseou-se na publicação da $\mathrm{FNB} \mathrm{WHO}^{5}$, com as adaptações necessárias para as condições locais.

Na África do Sul, em 1997, foi apresentado um conjunto de normas para promoção da saúde da população sul-africana de indivíduos maiores de 5 anos, uma vez que crianças menores necessitam de dietas específicas para o crescimento e desenvolvimento adequados ${ }^{18}$.

Os objetivos do grupo de pesquisa na elaboração do guia alimentar para sul-africanos foram: estabelecer o papel dos nutrientes e do padrão dietético da população; avaliar o entendimento e a aplicabilidade do guia alimentar; elaborar artigos científicos para divulgação; escrever as mensagens dos guias alimentares baseados nos alimentos com o intuito de atingir pessoas leigas e os profissionais de saúde; recomendar a forma de incorporação dos guias às políticas de saúde e de agricultura; aconselhar a implantação e a promoção do guia; desenvolver material de educação nutricional apropriado; monitorar o impacto no padrão dietético; adaptar o guia a diferentes grupos e contribuir para o processo de revisão a cada cinco anos ${ }^{18}$.

O guia sul-africano baseou-se em 10 mensagens claras e simples. Cada mensagem foi discutida durante o Congresso de Nutrição realizado em 1998, na cidade de Sun, com relação à sua relevância para a saúde, evidência científica, aplicação, compreensão e adequação ${ }^{19}$. Este guia foi revisado durante evento na Cidade de Cabo, em agosto de 1998. Em 1999, em Durban, foi realizado um encontro com o objetivo de desenvolver um protocolo para avaliar o guia alimentar.

Embora a África do Sul produza e exporte grande quantidade de alimentos, muitas famílias não têm acesso aos mesmos, especialmente na área rural, pois o fator socioeconômico é um dos principais aspectos que influenciam as escolhas alimentares e o padrão dietético na população africana ${ }^{19}$.

Em 1998, o Instituto de Pesquisa Alimentar da Europa realizou um estudo para verificar o padrão de consumo de alimentos e de nutrientes na população européia (Bélgica, Finlândia, Alemanha, Grécia, Irlanda, Itália, Holanda, Portugal, Espanha e Suécia) ${ }^{20}$. De acordo com Sandstrom ${ }^{20}$, o processo de desenvolvimento dos FBDG na Europa se deu em cinco etapas e a representação gráfica escolhida foi a pirâmide alimentar. A elaboração dos FBDG, na Europa, foi difícil devido às 
diferenças, tanto no padrão dietético como nos métodos para determinar o consumo alimentar dos indivíduos nos diversos países. Para o diagnóstico dos problemas de saúde relacionados à nutrição foram considerados aqueles mais comuns e uma das críticas na elaboração do guia alimentar na Europa foi o fato de que cada país deve elaborá-lo levando em consideração os problemas de saúde locais, bem como suas próprias recomendações nutricionais ${ }^{21}$. A partir da publicação do CINDI Dietary Guide ${ }^{22}$ alguns países da Europa desenvolveram seus próprios guias alimentares.

$\mathrm{Na}$ Espanha, em 1999, foi iniciado o processo de desenvolvimento da nova edição do guia alimentar, sendo realizado por pesquisadores da área da saúde e da sociedade científica. Diferentemente dos outros países, para a elaboração do guia cada grupo de pesquisadores analisou um grupo alimentar e em paralelo a composição dos alimentos, o valor nutricional, os aspectos culturais e antropológicos, a relevância socioeconômica, a tendência de consumo dos grupos alimentares, a seleção dos alimentos, as preparações habituais e seu impacto nas perdas dos nutrientes. Tais fatores constituem evidências científicas no desenvolvimento de fatores de risco e efeito protetor de cada grupo alimentar. Por fim, foram elaboradas 10 recomendações para dieta saudável (The Bilbao Declaracion, 2000). Baseado nas recomendações, o guia alimentar para população da Espanha foi resumido em uma representação gráfica no formato da pirâmide ${ }^{23}$.

Durante o desenvolvimento dos FBDG na Espanha deu-se destaque à importância dos aspectos culturais e antropológicos. De acordo com Barros $^{24} \mathrm{o}$ ato de comer não satisfaz apenas demandas biológicas, comungam com ele escolhas simbólicas calcadas na cultura da sociedade, sendo um dos aspectos apontados para o sucesso de políticas públicas de promoção da saúde.

Na Áustria, por não haver dados sobre o consumo alimentar da população, houve a adaptação das recomendações dietéticas da Alemanha e foram criadas as recomendações baseadas nos alimentos, intitulada "Dez regras para uma nutrição completa" (Ten Rules for a Wholesome Nutrition). Passados oito anos foi realizada pesquisa nacional abrangendo vários grupos populacionais (crianças, adolescentes, adultos e nutrizes), que revelou um comportamento nutricional semelhante ao da Alemanha e de outros países da Europa. Com isso não se estabeleceu outro FBDG, já que o proposto vinha sendo aceito pela população austríaca² .

No Brasil o primeiro guia alimentar desenvolvido foi para crianças menores de 2 anos em 2002, sendo iniciativa do Ministério da Saúde (Áreas Técnicas de Alimentação e Nutrição e da Saúde da Criança e Aleitamento Materno) e do Programa de Promoção e Proteção à Saúde da Organização Pan-Americana da Saúde (OPAS/Brasil) ${ }^{26}$.

Para a elaboração do guia alimentar o Ministério da Saúde (MS) designou o Departamento de Epidemiologia da Universidade de Pelotas (UFPel) como pólo de apoio técnico e de coordenação das atividades. Depois houve a identificação e a mobilização das instituições no âmbito estadual para o fornecimento de material bibliográfico e o subseqüente levantamento de dados sobre a situação alimentar e nutricional das crianças menores de 2 anos, com o objetivo de identificar os problemas comuns e as semelhanças e diferenças culturais existentes entre os estados ${ }^{27}$.

Realizou-se também um estudo qualitativo sobre percepções, práticas e tabus relacionados à alimentação da criança menor de 2 anos pelas Universidades Federais de Pelotas e do Rio Grande do Sul, com o objetivo de garantir que os resultados pudessem ser comparáveis entre as regiões e possibilitassem a sua utilização na elaboração de recomendações nacionais para o guia alimentar. $\mathrm{O}$ estudo foi realizado em cada macroregião e, posteriormente, juntaram-se todos os dados levantados para propor as recomendações para o Guia Alimentar Nacional, assim como definir sua representação gráfica e estrutura de publicação ${ }^{27}$.

De acordo com Fisberg et al. ${ }^{27}$, diferentemente do que tradicionalmente vinha sendo realizado no Brasil, buscou-se minimizar a verti- 
calização incluindo a participação dos profissionais de saúde do País e construindo as recomendações não apenas de forma a expressar o conhecimento científico adequado ao enfrentamento do problema populacional, mas também os aspectos culturais referentes às práticas da alimentação infantil revelados no estudo qualitativo nacional.

O guia alimentar para crianças brasileiras menores de 2 anos incluiu recomendações em forma de mensagens e por meio de uma representação gráfica. A figura escolhida foi a pirâmide alimentar que ilustra os três principais conceitos dos guias: a variedade, a moderação e a proporcionalidade ${ }^{27}$.

Também no Brasil, em 2004 foi desenvolvido o guia alimentar para população maior que 2 anos. Para sua elaboração realizou-se um levantamento do perfil de morbidade e mortalidade da população e de dados de consumo alimentar existentes no País e para sua implantação, até o presente momento, foi distribuída uma versão de bolso para a população ${ }^{28}$. Ressaltase que os dados de consumo alimentar também são antigos e podem não refletir mais a realidade da população brasileira, visto que foi publicada com base na Pesquisa de Orçamento Familiar ${ }^{29}$.

Com base no exposto acima se verifica que a elaboração dos guias alimentares abrange diversas etapas, sendo, portanto, um processo bastante complexo. Mesmo assim diversos países vêm se esforçando na construção de seus próprios guias alimentares, com o objetivo de melhorar os hábitos alimentares e a saúde de sua população.

\section{Implantação dos FBDG}

Após o desenvolvimento dos FBDG um dos grandes desafios é a sua implantação. Dentre as estratégias de comunicação dos FBDG destaca-se a elaboração de ferramentas de educação nutricional, tais como representação gráfica, folder, apostila e pôster. Pode-se obter o apoio das indústrias, das escolas, dos profissionais de saúde, dos centros de saúde, dos hospitais e da mídia para divulgação do material educativo. Atualmente, com o aumento do acesso da população à nova tecnologia de informação e comunicação podese divulgar os FBDG utilizando diversos websites. Para crianças podem-se adaptar as mensagens em músicas e jogos educativos ${ }^{21}$.

De acordo com Calderón \& Morón ${ }^{6}$, os FBDG devem ser incorporados aos programas escolares como iniciativa das escolas promotoras de saúde da Organização Pan-Americana de Saúde e da Organização Mundial de Saúde. Dessas atividades educativas devem participar docentes, estudantes e responsáveis. Além dos setores de saúde e educação, as indústrias de alimentos, os comerciantes, os meios de comunicação devem ser incluídos por terem grande influência nas condutas alimentares da população.

Na América Latina a participação da indústria de alimentos se destaca mais na divulgação dos FBDG do que em sua elaboração. Calderón \& Morón ${ }^{6}$ apontaram como causa da escassa participação da indústria, nesta etapa inicial, a falta de coerência entre os conteúdos das mensagens difundidas pelo guia e aqueles divulgados pelas indústrias. A indústria poderia participar da elaboração, da difusão do guia e contribuir com o financiamento para sua validação e implantação. Porém, seria necessário definir a dimensão das relações entre a indústria e os órgãos técnicos responsáveis pela elaboração dos guias, para minimizar as distorções na sua utilização.

Molina ${ }^{30}$ destacou que, para a implantação dos guias alimentares na América Central (Costa Rica, Guatemala, El Salvador, Honduras e Panamá), houve a necessidade de um trabalho integrado com todos os setores públicos e privados envolvidos na promoção de dietas e estilo de vida saudáveis da população. De acordo com a autora, também, faz-se necessário o conhecimento das políticas públicas que apóiem a implantação dos guias alimentares, para que eles sejam incluídos em diferentes programas de saúde pública e bem estar social.

Uma das estratégias para implantação dos FBDG na Bulgária e na República Checa foi a 
definição de grupos da população com consumo alimentar semelhante e a elaboração de mensagens de acordo com os objetivos do grupo, isto é, população geral, grupos de risco, como obesos, crianças, adolescentes, gestantes e idosos. $\mathrm{Na}$ Bulgária também foram desenvolvidos guias específicos para programas de saúde pública como prevenção de osteoporose, de deficiência de iodo e promoção do aleitamento materno ${ }^{31}$.

Na Hungria foram desenvolvidas duas estratégias para a implantação dos FBDG, o programa a curto prazo e o programa a longo prazo. No primeiro foram distribuídos pôsteres, calendários, livros com o guia completo e apostilas. $\mathrm{Na}$ segunda etapa foram realizadas palestras nas escolas para estudantes, responsáveis e professores e foi feita a divulgação do guia em websites da Internet ${ }^{31}$.

No Brasil, para implantação do guia alimentar para crianças, elaborou-se uma publicação denominada "Os dez passos para a alimentação saudável da criança menor de dois anos", direcionada aos profissionais da atenção básica, em particular ao Programa de Saúde da Família. O Ministério da Saúde também prevê a impressão de cartazes e folders com os 10 passos e a pirâmide alimentar infantil para disponibilização na rede de serviços do Sistema Único de Saúde (SUS) ${ }^{27}$. Além disso, o Ministério da Saúde solicita sistematicamente aos destinatários dos materiais educativos que os avaliem e enviem suas críticas e sugestões, porém não se tem dados sobre essa avaliação.

Apesar de se constituir em etapas importantes, a implantação do FBDG ainda precisa ser sistematizada para que, efetivamente, possa atingir a população à qual se destina.

\section{Avaliação dos guias alimentares}

De acordo com Molina ${ }^{30}$, os guias alimentares podem ser avaliados em três níveis: processo, efeitos e impacto. O primeiro nível de avaliação tem objetivo de comparar o que foi planejado com o que foi implantado. $\mathrm{O}$ segundo diz respeito ao conhecimento, à aplicação e ao entendimento dos guias alimentares pela população. E por último, a avaliação do impacto dos guias, objetiva observar a modificação do padrão de morbimortalidade e os estilos de vida da população ou grupo estudado após a utilização dos guias ${ }^{30}$.

Segundo com Calderón \& Morón ${ }^{6}$, os guias podem ser avaliados por métodos quantitativos, como consumo alimentar, prevalência de doenças, e qualitativos, como pesquisa de opinião, entrevistas e grupos coordenados.

Podem-se destacar algumas pesquisas que avaliaram o impacto do guia dietético na promoção de mudanças no hábito alimentar. Na Inglaterra, foram realizados dois estudos, o primeiro foi um estudo longitudinal que apontou que as informações sobre a composição dos alimentos divulgadas pelo FBDG, denominado "O Balanço Saudável", ajudaram os indivíduos a modificar os padrões dietéticos para alcançar seus objetivos nutricionais ${ }^{32}$. O segundo estudo mostrou, a partir dos dados da Pesquisa Nacional de Alimentos ${ }^{33}$, as mudanças anuais no consumo alimentar da população. Verificou-se que, a partir de 1986, quando o guia alimentar britânico foi desenvolvido, houve diminuição do consumo total de gorduras e de gorduras saturadas, bem como considerável parcela da população atendeu às recomendações nutricionais ${ }^{34}$.

Para testar o guia sul-africano foram realizadas discussões em grupos focais com mulheres de diferentes grupos étnicos da área rural e urbana em duas das nove províncias existentes. Os resultados demonstraram que os participantes compreenderam os guias dietéticos baseados nos alimentos e conseguiram planejar dietas de um dia utilizando este guia. Porém, as participantes não compreenderam algumas mensagens com relação a determinadas terminologias e conceitos, tais como "leguminosas", "alimentos de origem animal" e "lanches mais saudáveis"18.

Com relação ao guia alimentar americano e segundo Keenan et al. ${ }^{35}$, são escassas as pesquisas que abordem seu conhecimento e entendimento pelos consumidores. De acordo Achterberg ${ }^{36}$, 
somente quatro estudos avaliaram o conhecimento da segunda edição do guia alimentar americano publicado em 1985, sendo observado que os consumidores apresentavam dificuldades em interpretar o guia e gostariam que ele fosse mais prático e útil.

Desde então nenhum estudo americano foi realizado para avaliar as edições seguintes, publicadas em 1990 e 1995. Somente uma pesquisa avaliou a percepção dos consumidores sobre a representação gráfica e o conteúdo de três edições do guia alimentar anteriores ao ano de 1995. Foram realizados 12 grupos focais com adultos afro-americanos, beneficiários de um programa alimentar, e indivíduos com sobrepeso. Os resultados mostraram claramente que as informações dos guias alimentares eram muito amplas e necessitavam ser mais específicas, fornecendo aos consumidores uma orientação de como melhorar a qualidade da sua dieta ${ }^{35}$.

Em 2002, foi realizada uma pesquisa para avaliar o conhecimento e o entendimento dos consumidores americanos com relação às recomendações nutricionais, apresentadas no boletim do guia alimentar para americanos publicado em 1995. Participaram 400 adultos americanos da cidade de Minesota e os resultados mostraram que metade dos participantes desconhecia a publicação do guia dietético. A outra metade conhecia o documento, porém 30\% não sabiam o seu nome. O número de recomendações do guia recordado por pessoa foi, em média, de 2,5 das 13 recomendações. Somente $17 \%$ dos participantes lembravam-se da recomendação relacionada à gordura. Por fim, os participantes interpretaram corretamente a recomendação da atividade física ${ }^{35}$. Apesar do desenvolvimento do guia alimentar ao longo de oito décadas nos EUA, ainda há alta prevalência de obesidade na população ${ }^{37}$.

Na Finlândia foram realizadas pesquisas anuais sobre o consumo alimentar da população que são consideradas ferramentas para avaliar rapidamente tendências e intervenções. O principal sucesso da implantação dos FBDG foi um significante aumento no consumo de frutas e horta- liças nos últimos 20 anos e a substituição do leite integral por semi-desnatado e desnatado. Também se observou uma redução no consumo de sal com a diminuição do consumo de peixes e cogumelos salgados, além da cooperação da indústria de alimentos na redução de sódio nos alimentos e da legislação na determinação dos limites de sódio nos alimentos. Durante o período de 1972-1997 houve uma redução na prevalência da hipertensão arterial e das concentrações sanguíneas de colesterol na população e, conseqüentemente, uma redução da mortalidade por doença cardiovascular. A experiência na Finlândia mostrou que mudanças no padrão dietético da população são possíveis, porém requerem intervenção persistente e uma avaliação constante ${ }^{31,38}$.

O Brasil ainda não delineou uma estratégia nacional para a avaliação do guia alimentar para crianças menores de dois anos e seu impacto na saúde das crianças. Em 2005, uma pesquisa realizada no Rio Grande do Sul avaliou os efeitos da intervenção baseada na implantação dos "Dez Passos para uma Alimentação Saudável: guia alimentar para crianças menores de dois anos" 26 sobre as condições nutricionais e de saúde de lactentes em famílias de baixa renda. Estudou-se uma amostra randomizada de 200 recém-nascidos para o grupo intervenção e 300 para o grupo controle. O grupo intervenção recebeu orientações dietéticas durante 10 visitas domiciliares, realizadas nos primeiros 10 dias após o parto e depois mensalmente aos 6, 8, 10 e 12 meses. Os dois grupos (intervenção e controle) receberam visitas aos 6 e 12 meses para acompanhamento de rotina pelos seus pediatras ${ }^{39}$.

Os resultados mostraram que a intervenção mostrou-se efetiva na melhora de alguns aspectos da saúde da criança (aleitamento materno, práticas alimentares e morbidade), sendo evidente a necessidade da ampla aplicação desse programa na rede básica de saúde e na comunidade. Além disso, estes resultados sugerem que o programa de orientação dietética promoveu modificações positivas nas práticas alimentares e nas condições de saúde da criança, porém foi insuficiente para prevenir a anemia ferropriva ${ }^{39}$. 
Pode-se observar que, em relação ao FBDG, grandes esforços são concentrados na elaboração do guia alimentar e poucas são as estratégias de implantação e avaliação. Porém, faz-se necessária a avaliação tanto da compreensão do guia alimentar como da mudança de hábitos alimentares da população, pois esta é a principal forma de avaliar a eficácia dos guias alimentares sobre a saúde da população.

\section{Problemas metodológicos no desenvolvi- mento e na implantação dos FBDG}

Alguns aspectos metodológicos devem ser considerados na coleta e na interpretação dos dados dietéticos para o desenvolvimento dos FBDG, como o tipo de inquérito dietético utilizado na pesquisa de consumo alimentar, pois fornece diferentes níveis de precisão dos resultados ${ }^{20}$, bem como o número de dias de coleta do consumo alimentar, por influenciar nas variações individuais e, conseqüentemente, no consumo usual dos indivíduos ${ }^{20,40}$.

De acordo com Becker \& Welten ${ }^{41}$, algumas pesquisas de consumo alimentar indicam que 0 valor energético total da dieta encontra-se abaixo do recomendado, devido à diminuição do consumo durante a realização da pesquisa. Esses autores propõem a utilização do conceito "ponto de corte de valores" como forma de minimizar esse problema. Tal conceito é baseado na razão do consumo alimentar recomendado sobre o consumo alimentar observado. A razão maior que 1,00 indica que o consumo dietético está abaixo do recomendado, implicando em problemas para o desenvolvimento do FBDG, como um grande número de pessoas que não atendam à recomendação de macronutrientes e micronutrientes e de fibras alimentares. Esse problema foi detectado no desenvolvimento dos FBDG na Suécia e na Holanda.

Outro aspecto a ser considerado diz respeito às tabelas de composição química dos alimentos, que, apesar de úteis, representam uma ferramenta pouco precisa na avaliação do consumo dietético, e deve-se atentar para suas limitações ${ }^{20}$. De acordo com Leclercq et al. ${ }^{42}$, os principais problemas das tabelas são: a cobertura parcial de alimentos e nutrientes, a variabilidade analítica e a falta de exatidão na descrição dos itens alimentares. Diante disso, o uso inapropriado dessas tabelas pode dificultar a avaliação do consumo alimentar e sua relação com o aparecimento de doenças.

Assim como em sua elaboração, muitos problemas são apontados para a implantação dos FBDG. Florentino et al..$^{21}$ e Sandstrom ${ }^{20}$ destacaram o pouco interesse político, a falta de conhecimento em nutrição dos profissionais de saúde, a dificuldade de conscientizar os consumidores, a influência dos fatores ambientais e pessoais nas escolhas alimentares, a falta de cooperação da indústria para elaboração dos seus produtos, a dificuldade de acesso aos alimentos saudáveis, a dificuldade de motivar as mudanças do comportamento alimentar, a falta de integração da escola com o setor de saúde e a influência da mídia, às vezes, negativa no comportamento alimentar. Além disso, os autores também destacam a falta de avaliação da eficácia do programa de educação nutricional, bem como do material de divulgação, além da dificuldade de apoio político e financeiro para a promoção da educação nutricional.

\section{O N CLUS Ã O}

A elaboração dos guias alimentares se constitui em um processo bastante complexo desde seu desenvolvimento, até a implantação e a avaliação.

Diversos países vêm se esforçando na construção de seus próprios guias com o objetivo de melhorar os hábitos alimentares de sua população, porém o maior problema encontrado é a falta de dados de consumo alimentar da população atualizados, devido ao seu alto custo. Apesar disso, grandes esforços concentram-se na elaboração do guia alimentar e poucas são as estratégias de implantação e avaliação dos mesmos. 
O grande desafio após a elaboração dos guias é a sua implantação, que deverá ser sistemática e efetiva para atingir a população, porém, conforme se observou, são poucas as estratégias para a divulgação do guia alimentar. Muitos países têm dificuldade na divulgação do guia devido à falta de integração dos diversos setores como educação, saúde e, principalmente da indústria de alimentos. Como mostra o presente estudo, a América Central e a Finlândia conseguiram implantar um guia alimentar pelo trabalho integrado com todos os setores públicos e privados envolvidos na promoção de dietas.

Além disso, faz-se necessária a definição de estratégias de avaliação da compreensão do guia e da mudança do hábito alimentar da população, pois esta é a melhor forma de avaliar a eficácia dos guias sobre a saúde da população. Porém, verifica-se que em alguns países, como por exemplo, nos Estados Unidos, os indivíduos encontraram dificuldades no entendimento do guia alimentar devido ao fato de as informações serem muito amplas demonstrando a importância de avaliação constante. No Brasil ainda não se delineou uma estratégia nacional de avaliação do guia alimentar, mas uma pesquisa local já demonstrou que a intervenção foi efetiva na melhora de alguns aspectos da saúde da criança no Rio Grande do Sul.

Por fim, os guias alimentares podem ser utilizados na melhoria das condições de saúde da população, desde que sejam planejados de acordo com o grupo populacional eleito, e que se incluam estratégias adequadas de implantação e avaliação.

\section{COLABORADORES}

R.M.S. BARBOSA, L.G.T. COLARES e E.A. SOARES participaram da concepção, do levantamento bibliográfico, do desenvolvimento e da discussão do manuscrito.

\section{REFERÊ NCIAS}

1. International Food Information Council. The dietary guidance gap: consumers know they are supposed to do something-but are fruzzy on the details. Food Insight 2006 [cited 2006 Nov 6]. Available from: <http://www.ific.org/foodinsight>.

2. Food and Nutrition Board/World Health Organization. International Conference on Nutrition. World Declaration and Plan of Action for Nutrition, FAONHO. Rome: WHO; 1992.

3. Anderson GH, Zlotkin SH. Developing and implementing food-based dietary guidance for fat in the diets of children. Am J Nutr. 2000; 72(Suppl.5): 1404-9.

4. Painter J, Rah JH, Lee YK. Comparison of international food guide pictorial representations. J Am Diet Ass. 2002; 102(4)483-9.

5. Food and Nutrition Board/World Health Organization. Preparation and use of food based dietary guidelines. Geneva, 1996. Report of joint FAO/WHO consultation.

6. Calderón T, Morón C. La elaboración de guías alimentarias basadas en alimentos en países de América Latina. Food Agriculture Organization/ Instituto Internacional de Ciências de la Vida, 1998 [acceso 2004 jan 20]. Disponible en: <http://www. fao.org>

7. Society for Nutrition Education. Comments on the center for nutrition policy and promotion: notice of proposal for food guide graphic presentation and consumer education materials. Fed Regist. 2004; 69(133):1-17.

8. Lanzillotti HS, Couto SEM, Affonso FM. Pirâmides alimentares: uma leitura semiótica. Rev Nutr. 2005; 18(6):785-92.

9. Health Education Authority. Proposals for the development of national food selection guide for the United Kingdom: background paper. Prepared by Issy Cole-Hamilton, for an Internal HEA. Report. London; 1991.

10. Myers EF, Britten P, Davis CA. Past, present, and future of the food guide pyramid. J Am Diet Assoc. 2001; 101(8):881-5.

11. Hunt P, Rayner M, Gatenby S. A national food guide for UK. Background and development. J Hum Nutr Diet. 1995; 8(5):315-22.

12. Peña M. Guias de alimentación en América Latina. Anais do $1^{\circ}$ Workshop Instituto Danone. Alimentação equilibrada para a população brasileira; 1998, Florianópolis. Florianópolis: Instituto Danone; 1998. p.31-43.

13. Nestle M. In defense of the USDA Food Guide Pyramid. Nutr Today. 1998; 33(5):189-92.

14. Achterberg C. Development of FBDG: collection and use of data. Workshop on National Food Based Dietary Guidelines: experiences, implications and future directions; April 28-30; Budapest: FAO; 2004. 
15. Jiménez $A$, Jones $E$, Bacardí $M$. La manzana de la salud. Divulgare. 1996; 14:12-1

16. Bacardi-Gascon M, Jiménez-Cruz A, Jones E. An evaluation of two Mexican food guides. Int J Food Sci Nutr. 2002; 53(2):163-9.

17. Rodrigues SSP, Franchini B, Graça P, Almeida MDV. A new food guide for the portuguese population: development and technical considerations. J Nutr Educ Behav. 2006; 38(3):189-95.

18. Vorster HH, Love P, Browne. Development of foodbased dietary guidelines for South Africa: the process. S Afr J Clin Nutr. 2001; 14(3):3-6.

19. Love P, Maunder E, Green M, Ross F, Smale-Lovely J, Charlton K. South African food-based dietary guidelines: testing of the preliminary guidelines among women in KwaZulu-Natal and the Western Cape. S Afr J Clin Nutr. 2001; 14(1):9-19.

20. Sandstrom B. A framework for food-based dietary guidelines in the European Union working party 2: final report. Public Health Nutr. 2001; 4(2A): 293-305.

21. Florentino R, Tee ES, Poh BK. Report of a seminar and workshop on food vased dietary guidelines and nutrition education: bridging science and communication. Asia Pac J Clin Nutr. 1999; 8(4): 291-9.

22. World Health Organization. CINDI dietary guide. Geneve: WHO; 2001.

23. Aracenta J, Serra-Manjem L. Dietary guidelines for the Spanish population. Public Health. 2001; 4(6A): 1403-8.

24. Barros DC. O consumo alimentar de gestantes adolescentes no Município do Rio de Janeiro [dissertação]. Rio de Janeiro: Fundação Oswaldo Cruz; 2002.

25. Koeing J, Elmadfa I. Food-based guidelines: the Austrian perspective. Br J Nutr. 1999; 81(Suppl 2): 31-5.

26. Brasil. Ministério da Saúde. Dez passos para uma alimentação saudável: guia alimentar para crianças menores de dois anos. Brasília: Ministério da Saúde/Organização Pan-Americana da Saúde; 2002.

27. Fisberg RM, Slater B, Marchioni DML, Martini LA. Inquéritos alimentares: métodos e bases científicas. Barueri: Manole; 2005.

28. Brasil. Ministério da Saúde. Guia alimentar para a população brasileira. Promovendo alimentação saudável. Brasília; 2006

29. Instituto Brasileiro de Geografia e Estatística. Pesquisa de orçamentos familiares, 1995 -1996. Primeiros resultados. Rio de Janeiro; 1997.
30. Molina V. Guias alimentarias en Centro America. Panamá: Instituto de Nutrición de Centro America y Panamá; 1999.

31. International Life Science Institute. National Food Based Dietary Guidelines: Experiences, Implications and Future Directions. Summary Report of a Workshop. 2004.

32. Patterson RE, Kristal AR, White E. Do beliefs, knowledge and perceived norms about diet and cancer predict dietary change? Am J Public Health. 1996; 86(10):1394-400.

33. Ministry of Agriculture, Fisheries and Food. The dietary and nutrition survey of British adults further analysis. London: HMSO; 1994.

34. Wearne ST, Day MJL. Clues for the development of food-based dietary guidelines: how are dietary targets being achieved by UK consumers? Br J Nutr. 1999; 81(Suppl. 2):119-26.

35. Keenan DP, Abusabha R, Robinson NG. Consumers' understanding of the dietary guidelines for americans: insights into the future. Health Educ Behav. 2002; 29(1):124-35.

36. Achterberg CL. Qualitative research: what do we know about teaching good nutritional habits? J Nutr. 1994; 124(Suppl 9):1808-12.

37. Baskin ML, Ard J, Franklin F, Allison DB. Prevalence of obesity in the United States. Obes Rev. 2005; 6(1):5-7.

38. Pietinen P, Lahti-Koski M, Vartiainen E, Puska P. Nutrition and cardiovascular disease in Finland since the early 1970s: a success story. J Nutr Health Aging. 2001; 5(3):150-4.

39. Vitolo MR, Bortolini GA, Feldens CA, Drachle ML. Impactos da implementação dos dez passos da alimentação saudável para crianças: ensaio de campo randomizado. Cad Saúde Pública. 2005; 21(5):1448-57.

40. Lambe J, Kearney J. The influence of survey duration on estimates of food intakes relevance for food-based dietary guidelines. Br J Nutr. 1999; 81(Suppl 2):139-42

41. Becker W, Welten D. Under-reporting in dietary surveys: implications for development of foodbased dietary guidelines. Public Health Nutr. 2001; 4(2B):683-7.

42. Leclercq C, Valsta LM, Turrini A. A food composition issues-implications for the development of food-based dietary guidelines. Public Health Nutr. 2001; 4(2B):677-82.

Recebido em: 8/3/2007

Versão final reapresentada em: 11/3/2008 Aprovado em: 18/3/2008 